\title{
Activation Volume for Arsenic Diffusion in Germanium
}

\section{Citation}

Mitha, Salman, Michael J. Aziz, David Schiferl, and David B. Poker. 1996. Activation volume for arsenic diffusion in germanium. Applied Physics Letters 69(7): 922-924.

\section{Published Version}

http://dx.doi.org/10.1063/1.116944

\section{Permanent link}

http://nrs.harvard.edu/urn-3:HUL.InstRepos:2959838

\section{Terms of Use}

This article was downloaded from Harvard University's DASH repository, and is made available under the terms and conditions applicable to Other Posted Material, as set forth at http:// nrs.harvard.edu/urn-3:HUL.InstRepos:dash.current.terms-of-use\#LAA

\section{Share Your Story}

The Harvard community has made this article openly available.

Please share how this access benefits you. Submit a story.

\section{Accessibility}




\title{
Activation volume for arsenic diffusion in germanium
}

\author{
Salman Mitha ${ }^{\mathrm{a})}$ and Michael J. Aziz ${ }^{\mathrm{b})}$ \\ Division of Applied Sciences, Harvard University, Cambridge, Massachusetts 02138 \\ David Schiferl \\ Los Alamos National Laboratory, Los Alamos, New Mexico 87545 \\ David B. Poker \\ Solid State Division, Oak Ridge National Laboratory, Oak Ridge, Tennessee 38731
}

(Received 3 May 1996; accepted for publication 10 June 1996)

\begin{abstract}
We have measured the effect of pressure on As diffusion in Ge. Diffusion anneals on ion-implanted samples were carried out in a high-temperature diamond anvil cell using fluid argon as a clean, hydrostatic pressure medium. At $575^{\circ} \mathrm{C}$ over the pressure range $0.1-4 \mathrm{GPa}$, pressure slightly enhances the diffusivity, characterized by an activation volume of $-1.7 \pm 1.4 \mathrm{~cm}^{3} / \mathrm{mole}$ or $-0.12 \pm 0.10$ times the atomic volume. The results call into question the prevailing view that diffusion of groups III, IV, and V elements are mediated entirely by vacancies. If diffusion of As is mediated entirely by vacancies then either the vacancy formation volume must be unexpectedly low or the energy of vacancy migration must be unexpectedly high. (C) 1996 American Institute of Physics. [S0003-6951(96)02132-8]
\end{abstract}

In the elemental solids the diffusion mechanism is an intrinsic property of fundamental scientific interest. Because understanding and controlling diffusion related phenomena become increasingly important as semiconductor device dimensions decrease, diffusion in semiconductors has been heavily studied. Despite this emphasis, there remains no consensus about the relative roles of the various proposed mechanisms for diffusion of many group III, IV, and V elements in Si. Diffusion of these elements in Ge is commonly believed to be mediated entirely by vacancies, but this perception is based on few experimental studies. ${ }^{1,2}$ The increasing importance of $\mathrm{Ge}$ for uses such as in $\mathrm{Si}_{1-x} \mathrm{Ge}_{x}$ devices necessitates further study of diffusion in Ge.

The effect of pressure, $p$, on the diffusivity, $D$, is characterized by the activation volume, $\Delta V^{*}$, according to

$$
\Delta V^{*}=-k T\left(\frac{\partial \ln D}{\partial p}\right)_{T},
$$

when negligible correction terms are neglected. ${ }^{3} \Delta V^{*}$ can be either positive or negative, depending upon whether $D$ decreases or increases with $p$, respectively. $\Delta V^{*}$ is the sum of two components

$$
\Delta V^{*}=\Delta V_{f}+\Delta V_{m},
$$

where $\Delta V_{f}$, the formation volume, is the volume change in the system upon formation of one defect in its standard state, and $\Delta V_{m}$, the migration volume, is the additional volume change when the defect reaches the saddle point in its migration path. $\Delta V_{f}$ characterizes the pressure dependence of the equilibrium point defect concentration while $\Delta V_{f}$ characterizes the pressure dependence of the defect mobility.

Unlike activation energies, which are always positive, activation volumes are expected to have different signs for different types of point defect mechanisms. Hence, a mea-

\footnotetext{
${ }^{a)}$ Present address: Charles Evans and Associates, 301 Chesapeake Dr., Redwood City, CA 94063.

b)Electronic mail: aziz@das.harvard.edu
}

surement of the activation volume can be a more revealing test for distinguishing between potential diffusion mechanisms. The only published measurement of $\Delta V^{*}$ for diffusion in $\mathrm{Ge}$ is that of Werner et al. for self-diffusion. ${ }^{4}$ In silicon there are more such measurements. ${ }^{5-7}$

Samples were prepared by ion implantation of ${ }^{75} \mathrm{As}^{+}$at $500 \mathrm{keV}$ to a dose of $2 \times 10^{14} / \mathrm{cm}^{2}$ into $\mathrm{Ge}(100)$ wafers 50 $\mu \mathrm{m}$ thick at $77 \mathrm{~K}$. Wafers were subsequently implanted with ${ }^{71} \mathrm{Ge}^{+}$at 250 and $500 \mathrm{keV}$ to doses of 5 and $8 \times 10^{14} / \mathrm{cm}^{2}$, respectively, at $77 \mathrm{~K}$ to amorphize the implanted layer which is necessary for the subsequent restoration of defect-free crystal by solid phase epitaxial growth (SPEG). SPEG occurs at temperatures too low for measurable diffusion to occur; $\sim 15$ min anneals at $450{ }^{\circ} \mathrm{C}$ restores crystallinity in our samples. Samples are then cleaved into small pieces $\sim 150$ $\mu \mathrm{m}$ by $150 \mu \mathrm{m}$ to fit into the diamond anvil cell (DAC).

Diffusion anneals were carried out for various pressures at $575^{\circ} \mathrm{C}$ for 1800 or $4200 \mathrm{~s}$. The high pressure device is a modified Merrill-Bassett DAC, ${ }^{8}$ loaded cryogenically with liquid $\mathrm{Ar}$ as a pressure transmitting medium. Two types of gaskets, rhenium and inconel X750 were used. The cell is externally heated in an inert atmosphere in a furnace.

Pressure is measured using fluorescence peak shifts of Sm doped YAG (Sm:YAG). ${ }^{9}$ Pressure was determined by simultaneously fitting the 617,616 , and $610 \mathrm{~nm}$ lines. The fit is based on Hess and Schiferl's calibration of the 617 and $616 \mathrm{~nm}$ lines. The pressure coefficient of the $610 \mathrm{~nm}$ line was calibrated against the other two at ambient temperature. Then by assuming that the temperature shifts are negligible, as Hess and Schiferl found for the other two lines, we developed a protocol for fitting all three lines. This procedure was necessary for robust and repeatable fitting.

Diffusion is measured ex situ by sputter sectioning using secondary ion mass spectrometry (SIMS). We used a VG Ionex $1170 \mathrm{X}$ magnetic sector SIMS. The primary beam was $16 \mathrm{keV} \mathrm{Cs}^{+}$, rastered to produce a flat crater bottom. To remove crater wall effects secondary ions were collected from an area that was gated electronically to cover only the 


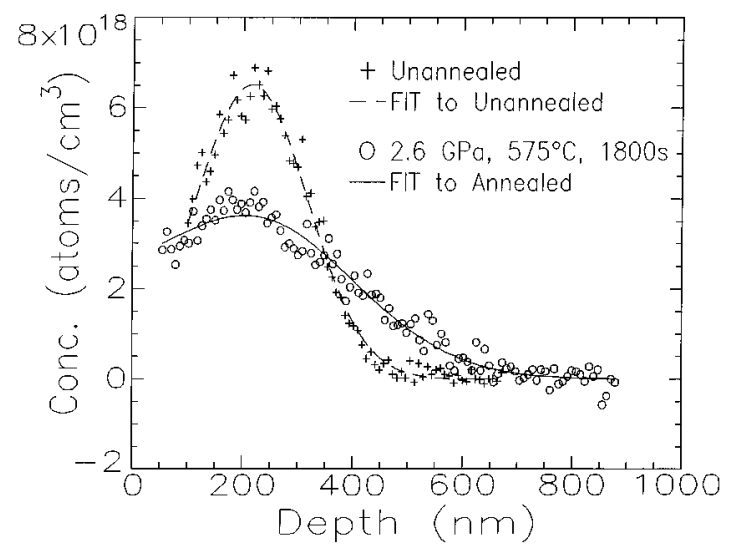

FIG. 1. Depth profiles and Gaussian fits.

central $5 \%$ of the area of the crater. The $\mathrm{AsGe}^{-}$ion was tracked for the As concentration profile. The implanted depth profile is Gaussian within our experimental resolution. The top $100 \mathrm{~nm}$ of the profiles are lost due to a surface transient artifact of SIMS. The amount of diffusion was determined by fitting Gaussians to the broadened depth profiles, using image profiles at negative depth to account for the reflecting wall at the surface. Figure 1 shows typical profiles.

Using Eq. (1) $\Delta V^{*}$ was calculated by fitting a straight line to the points in Fig. 2. The result is $\Delta V^{*}=-1.7$ $\pm 1.4 \mathrm{~cm}^{3} / \mathrm{mole}=-0.12 \pm 0.10 \Omega$, where $\Omega$ is the atomic volume of $\mathrm{Ge}$ at standard $T$ and $p$. The fit was performed using a weighted linear least-squares technique. For comparison, slopes for activation volumes of $\pm 0.5 \Omega$ and \pm 1.0 $\Omega$ are also shown in Fig. 2. The intercept, $1.3 \times 10^{-14} \mathrm{~cm}^{2} / \mathrm{s}$, is the ambient pressure diffusivity of As in $\mathrm{Ge}$ at $575^{\circ} \mathrm{C}$ and it agrees well with the value of 1.0 $\times 10^{-14} \mathrm{~cm}^{2} / \mathrm{s}$ calculated from the published Arrhenius parameters. $^{10}$

If diffusion is point defect mediated, Eq. (2) is valid only if the point defect concentrations maintain their equilibrium levels for the pressures and temperatures of the experiment. We have shown this to be the case for this experiment. ${ }^{11}$

In Table I we compare our measured $\Delta V^{*}$ with that of Werner et al. $^{4}$ for Ge self-diffusion under various conditions and those of Lu et al. ${ }^{12}$ for SPEG in Ge. By measuring the effects of dopants on self-diffusion and modeling the dependence of the charged vacancy concentration on the Fermi

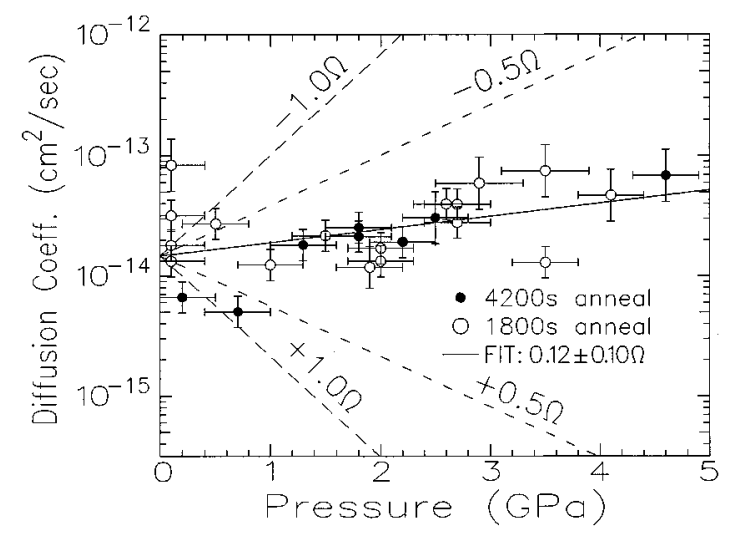

FIG. 2. Activation volume plot at $575^{\circ} \mathrm{C}$.
TABLE I. Activation volumes in germanium.

\begin{tabular}{lccc}
\hline \multicolumn{1}{c}{ Process } & $\Delta V^{*}(\Omega)$ & $T\left({ }^{\circ} \mathrm{C}\right)$ & Authors \\
\hline As diffusion in Ge & $-0.12 \pm 0.1$ & 575 & this work \\
SPEG in Ge & -0.46 & 350 & Lu et al. \\
Self-diffusion in Ge & +0.24 & 603 & Werner et al. \\
Self-diffusion in Ge & +0.41 & 813 & Werner et al. \\
Self-diffusion by $V^{-}$ & +0.28 & 700 & Werner et al. \\
Self-diffusion by $V^{0}$ & +0.56 & 700 & Werner et al. \\
\hline \hline
\end{tabular}

level (assuming all other parameters, such as jump rates and activation volumes are invariant), Werner et al. also determined that the negative vacancy is responsible for $77 \%$ of the transport for self-diffusion in $\mathrm{Ge}$ at $700{ }^{\circ} \mathrm{C}$.

We now discuss the implications of these results for possible diffusion mechanisms. We first show that it is difficult to reconcile the simple vacancy mechanism with the measurements. We then discuss other possible mechanisms, although these data do not permit a critical test of them.

Let us consider the possibilities for the relative contributions through Eq. (2) of formation and migration terms to the measured activation volume for a $100 \%$ vacancy mechanism. Werner et al. assumed that $\Delta V_{m}$ is small and used $\Delta V^{*}$ as an approximation for $\Delta V_{f}$. With this they concluded that the vacancy is the predominant point defect mediating self diffusion. If we assume $\Delta V_{m}$ to be negligible, our results imply $\Delta V_{f}=-0.12 \Omega$. This value would be consistent with a direct interchange mechanism ${ }^{13}$ because it does not involve a point defect and consequently $\Delta V_{f}=0$. Werner et al. base their assumption of small $\Delta V_{m}$ on studies of self-diffusion in gold. ${ }^{14}$ The assumption may not be valid for Ge.

Lu et al. measured large negative $\Delta V^{*}=-0.46 \Omega$ for SPEG and suggested that their value is approximately equal to $\Delta V_{m}$. If we assume that the atomic transport processes of SPEG and diffusion in tetrahedral covalent networks have similar $\Delta V_{m}$, values as large and negative as $\Delta V_{m}=$ $-0.4 \Omega_{\mathrm{Ge}}$ are then plausible for diffusion in Ge. In this case $\Delta V_{f}=+0.3 \Omega$. Such a small $\Delta V_{f}$ implies a very large inward relaxation around the vacancy. For comparison, first principles calculations ${ }^{15}$ for $\mathrm{Si}$ give a formation volume of $+0.75 \Omega_{\mathrm{Si}}$ and we currently have no reason to expect a large difference between $\Delta V_{f}$ in $\mathrm{Si}$ and $\mathrm{Ge}$.

Finally if we assume $\Delta V_{f}=+0.75 \Omega$ in accordance with the theoretical result for Si then our experiment implies $\Delta V_{m}=-0.87 \Omega$. The difference between the enthalpy of migration at zero pressure $\left(\Delta E_{m}\right)$ and high pressure $\left(\Delta H_{m}\right)$ is $p \Delta V_{m} \cdot{ }^{16}$ In $\mathrm{Si}, \Delta E_{m}$ for vacancies ranges from 0.18 to $0.45 \mathrm{eV}$ for different charge states. ${ }^{17-20}$ Scaling with the bond strength ratios gives expected $\Delta E_{m}$ in Ge of $0.14-$ $0.36 \mathrm{eV}$. When $p \Delta V_{m}$ is added to these values, the migration enthalpy vanishes at a critical pressure of $p_{\text {crit }}=\Delta E_{m} / \Delta V_{m}$ $=1.2-3.0 \mathrm{GPa}$. At $p_{\text {crit }}$ we expect some kind of break in the slope in Fig. 2 as the barrier to migration vanishes, precluding a further reduction in the barrier height with increasing $p$. Such a break is not observed.

Figure 3 graphically summarizes these arguments. The permitted combinations of $\Delta V_{f}$ and $\Delta V_{m}$ lie within the diagonal band. Assuming $\Delta E_{m}<0.36 \mathrm{eV}$ and, from Fig. 2, $p_{\text {crit }}>3.5 \mathrm{GPa}$ requires that $\Delta V_{m}>-0.73 \Omega$. Thus we exclude the cross-hatched region below the horizontal line. The 


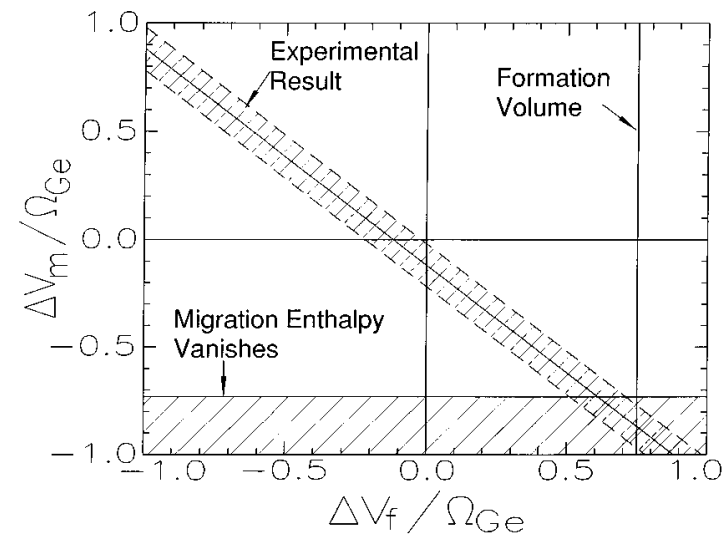

FIG. 3. Permitted values of $\Delta V_{f}$ and $\Delta V_{m}$ for simple vacancy mechanism.

expected formation volume, indicated by the vertical line, intersects the diagonal band in the excluded region. If diffusion is mediated entirely by vacancies then either the vacancy formation volume must be lower than expected or the migration energy must be greater than expected. Experimental studies are currently under way to raise the lower limiting value of $p_{\text {crit }}$ in Fig. 2, which would in turn raise the horizontal line bounding the excluded region in Fig. 3.

Multiple diffusion mechanisms may be operating in parallel. By comparing our data with calculated activation plots for a pair of diffusion mechanisms operating in parallel we have determined that our data are inconsistent with a double defect mechanism where the activation volumes are large and of opposite signs. ${ }^{11}$ Two mechanisms, however, with opposite-signed activation volumes of magnitude less than about $0.4 \Omega$ cannot be ruled out by the data.

The large positive $\Delta V^{*}$ of Werner et al. is the principal evidence for the predominance of the vacancy mechanism for self-diffusion in Ge. They also show that the negative vacancy, $V^{-}$, is predominant. Donors such as As should be positively charged and should bind to $V^{-}$. (This is indeed the case in $\mathrm{Si}^{1}{ }^{1,21}$ ) The much faster diffusion of donors than acceptor and self-diffusion in $\mathrm{Ge}^{1}$ has been attributed to $\mathrm{As}^{+}-V^{-}$association. ${ }^{2,22}$ Because donors raise the Fermi level, the predominance of $V^{-}$is further enhanced. However, in diamond cubic structures the increased association of a vacancy with an impurity atom due to binding may not cause a large increase in the impurity diffusivity by the normal vacancy mechanism, because the pair must dissociate in order to migrate: binding therefore causes an offsetting reduction in the correlation factor. ${ }^{23} \mathrm{We}$ note that this reduced correlation factor does not apply to delocalized or "extended" vacancies ${ }^{24}$ or if significant binding still exists between vacancy and impurity in third-neighbor locations. ${ }^{23}$ It is not yet known into which category the case at hand falls.

We now consider other mechanisms. If the interstitialcy mechanism predominates for arsenic diffusion then there must be some explanation why it is not the predominant mechanism for self-diffusion. One suggestion due to Frank ${ }^{25}$ is that negative interstitials may be mediating As diffusion in Ge. Association with negative interstitials could then enhance significantly the diffusion of donors compared to selfdiffusion and also explain a nonpositive $\Delta V^{*}$.

It is also possible that a vacancy mechanism is operating with jumps between lattice sites that are not nearest neighbors. Second-neighbor vacancy jumps have been proposed to occur in GaAs. ${ }^{26}$ In Ge, second-neighbor jumps of As or Ge would permit long-range transport of As without $\mathrm{As}^{+}-V^{-}$dissociation, and ball-and-stick modeling of the midpoint of the migration path indicates that a large negative migration volume is plausible. ${ }^{27}$ However, calculations of the energetics and volumetrics involved are required to check the feasibility of such mechanisms. Of course, a more complex mechanism may be operating.

In summary, the small, negative activation volume for As diffusion calls into question the prevailing view that diffusion of group III, IV, and V elements is mediated entirely by a normal vacancy mechanism in Ge. If diffusion of As is mediated entirely by vacancies then either the vacancy formation volume is unexpectedly low or the energy of vacancy migration is unexpectedly high. The interstitialcy and direct interchange mechanisms cannot be ruled out. Accurate calculations of the energetics and volumetrics of formation and migration of various defects would be very helpful.

We are indebted to the late John Martin of MIT Materials Characterization Facilities for his assistance with SIMS. This research was supported by NSF-DMR-95-25907. Work at ORNL and LANL was supported by the Division of Materials Sciences, DOE under DE-AC05-96OR22464 and W-7405-ENG-36 with Lockheed Martin Energy Research Corp. and the University of California, respectively.

${ }^{1}$ W. Frank, U. Gosele, H. Mehrer, and H. Seeger, in Diffusion in Crystalline Solids, edited by G. E. Murch and A. S. Norwick (Academic, Orlando, FL, 1984), p. 63.

${ }^{2}$ W. Frank, in Crucial Issues in Semiconductor Materials and Processing Technologies, edited by S. Coffa (Kluwer, The Netherlands, 1992), p. 383.

${ }^{3}$ R. N. Jefferey and D. Lazarus, J. Appl. Phys. 41, 3186 (1970).

${ }^{4}$ M. Werner, H. Mehrer, and H. D. Hochheimer, Phys. Rev. B 32, 3930 (1985).

${ }^{5}$ E. Nygren, M. J. Aziz, D. Turnbull, J. M. Poate, D. C. Jacobson, and R. Hull, Appl. Phys. Lett. 47, 105 (1985).

${ }^{6}$ M. J. Aziz, E. Nygren, W. H. Christie, C. W. White, and D. Turnbull, Mater. Res. Soc. Symp. Proc. 36, 101 (1985).

${ }^{7}$ U. Södervall, M. Friesel, and A. Lodding, J. Chem. Soc. Faraday Trans. 86, 1293 (1990).

${ }^{8}$ L. Merrill and W. A. Bassett, Rev. Sci. Instrum. 45, 290 (1974).

${ }^{9}$ N. Hess and D. Schiferl, J. Appl. Phys. 71, 2082 (1992).

${ }^{10}$ W. C. Dunlap, Jr., Phys. Rev. 94, 1531 (1954).

${ }^{11}$ S. Mitha, Phd.D. thesis, Harvard University, 1995.

${ }^{12}$ G. Q. Lu, E. Nygren, and M. J. Aziz, J. Appl. Phys. 70, 5323 (1991).

${ }^{13}$ K. C. Pandey, Phys. Rev. Lett. 57, 2287 (1986).

${ }^{14}$ R. M. Emrick, Phys. Rev. 122, 1720 (1961).

${ }^{15}$ A. Antonelli and J. Bernholc, Phys. Rev. B 40, 10643 (1989).

${ }^{16}$ We assume $d\left(\Delta V_{m}\right) / d p$ to be negligible.

${ }^{17}$ P. M. Fahey, P. B. Griffin, and J. D. Plummer, Rev. Mod. Phys. 61, 289 (1989).

${ }^{18}$ G. D. Watkins, Inst. Phys. Conf. Ser. 23, 1 (1974).

${ }^{19}$ G. D. Watkins, J. R. Troxwell, and A. P. Chatterjee, Inst. Phys. Conf. Ser. 46, 16 (1978).

${ }^{20}$ J. L. Newton, A. P. Chatterjee, R. D. Harris, and G. D. Watkins, Physica (Utrecht), 116B, 219 (1983).

${ }^{21}$ M. Hirata, M. Hirata, and H. Saito, J. Phys. Soc. Jpn. 27, 405 (1969).

${ }^{22}$ R. J. Borg and G. J. Dienes, An Introduction to Solid State Diffusion (Academic, San Diego, 1988), p. 196.

${ }^{23}$ S. M. Hu, Phys. Status Solidi B 60, 595 (1973).

${ }^{24}$ A. Seeger and K. P. Chik, Phys. Status Solidi 29, 455 (1968).

${ }^{25} \mathrm{~W}$. Frank (private communication).

${ }^{26}$ D. Shaw, Phys. Status Solidi A 86, 629 (1984).

${ }^{27}$ E. Kaxiras and M. J. Aziz (unpublished). 